\title{
Biolaminóides Calcários Holocênicos da Lagoa Vermelha, Brasil
}

Holocenic Calcareous Biolaminoids of the Vermelha Lagoon, Brazil

\author{
Loreine Hermida da Silva e Silva* \& \\ Sinda Beatriz Vianna Carvalhal** \\ * Departamento de Ciências Naturais. Universidade Federal do Estado do Rio de Janeiro \\ (UNIRIO). Avenida Pasteur $n^{\circ}$ 458, laboratório 409. Urca, Rio de Janeiro, RJ, Brasil. \\ 22.290-240.e-mail: loreineh@unirio.br \\ **Programa de Pós Graduação em Geologia. Instituto de Geociências. Universidade do \\ Brasil (UFRJ). 21.949-900. Rio de Janeiro, RJ, Brasil. \\ e-mail: vcarvallis@ig.com.br \\ Recebido em: 02/10/2005 Aprovado em: 08/10/2005
}

\section{Resumo}

A Lagoa Vermelha faz parte do complexo lagunar de Araruama e está situada entre as coordenadas $22^{\circ} 55^{\prime} 39^{\prime \prime}$ e $22^{\circ} 56^{\prime} 06^{\prime \prime}$ de latitude Sul e $42^{\circ} 21^{\prime} 29^{\prime \prime}$ e $42^{\circ} 24^{\prime} 13^{\prime \prime}$ de longitude Oeste de Greenwich. Suas dimensões são de 4.400 $\mathrm{m}$ de comprimento, 250-850 m de largura com uma área de aproximadamente $2.400 \mathrm{~m}^{2}$ e profundidade de 0,2 a $1,7 \mathrm{~m}$. Este estudo se baseou em coletas realizadas ao longo de cinco estações localizadas na margem nordeste da lagoa, onde foram retiradas 20 amostras de biolaminóides. Para a caracterização da composição microbiana dos tipos de biolaminóides foram confeccionadas lâminas frescas e permanentes e a sua análise foi realizada em microscopia. Foram encontrados três tipos de biolaminóides sendo eles liso, poligonal e pustular. Os biolaminóides liso e poligonal apresentaram estratificações enquanto o pustular apresentou-se homogêneo. Tanto os biolaminóides estratificados quanto o homogêneo mostraram-se compostos por um tipo principal de cianobactéria formadora; no entanto, os biolaminóides estratificados apresentaram na camada superficial a predominância das espécies filamentosas e nas camadas mais profundas o predomínio das cocóides. A análise dos biolaminóides encontrados revelou um total de 33 espécies de cianobactérias, comprovando que essas estruturas têm como seu 
agente formador principal estas microalgas. O biolaminóide liso revelou ser formado principalmente por Lyngbya aestuarii, o biolaminóide poligonal exibiuse composto por Microcoleus chthonoplastes e o pustular caracterizou-se pela presença da cianobactéria Chlorogoea tuberculosa. O predomínio das cianobactérias se justifica pelo fato destas serem portadoras de alta plasticidade morfológica, o que lhes permite sobreviver em vários ambientes e predominar nos hipersalinos.

Palavras-Chave: biolaminóides, cianobactérias, estromatólitos, ambientes hipersalinos, lagoa Vermelha.

\section{Abstract}

Lagoa Vermelha lies within the coordinates 22 $55^{\prime} 39^{\prime \prime}-22^{\circ} 56^{\prime} 06^{\prime \prime} \mathrm{S}$ and $42^{\circ} 21^{\prime \prime} 29^{\prime \prime}-42^{\circ} 24^{\prime} 13^{\prime \prime} \mathrm{W}$ and is part of Araruama lagoon complex. Its dimensions are 4,400 $\mathrm{m}$ in length, $250-850 \mathrm{~m}$ in width, with approximate area of 2,400 $\mathrm{m}^{2}$ and $0,2-1,7 \mathrm{~m}$ in depth. The study was based on six monthly collections in five stations over the north - east lagoon's margins from where was taken 20 samples of biolaminoids. To determinate the microbial composition of all different kinds of biolamonoids was made fresh and permanent slides and its analysis was carried out by microscopy. It was found three different kinds of microbial mats distributed throughout all five stations and were called: flat, polygonal and pustuled. Flat and polygonal microbial mats show itself layered, while pustuled show homogeneous structures. Layered and homogeneous ones present the same kind of main cyanobacterias, however layered mats in superficial stratum show preponderance of filamentous species and coccoid species in the bottom. Analysis showed a total of 33 species of cyanobacterias confirming it as main biolaminod component.The flat biolaminoid was mainly formed by Lyngbya aestuarii, the polygonal biolaminoid showed itself composed by Microcoleus chthonoplastes and the pustular biolaminoid was characterized by the presence of the cyanobacteria Chlorogoea tuberculosa. The cyanobacterial dominance is justified through its high morphologic adaptations that ensure its survival in many environments and prevail over in hipersaline local.

Key words: biolaminoids, cyanobacteria, stromatolites, hipersaline environment, Vermelha lagoon.

\section{Introdução}

Os estromatólitos são considerados as mais antigas evidências macroscópicas de vida na Terra e são encontrados em todos os continentes, 
principalmente em rochas do Pré-Cambriano, período considerado como a "Era dos Estromatólitos”. Atualmente são definidos como construções organosedimentares produzidas pelo aprisionamento ou pela captura e precipitação de sedimentos, resultando fundamentalmente da atividade metabólica de microorganismos principalmente as cianobactérias (Silva e Silva, 2002). Ocorrem, predominantemente, em ambientes deposicionais de carbonato, com maior número de ocorrências em dolomitos e calcários, sendo menos comuns em sedimentos clásticos terrígenos, rochas silicosas, fosfatos, gipsita e em lugares associados com depósitos de ferro. Grande parte dos estromatólitos carbonáticos é formada em águas marginais rasas de bacias marinhas e lagos salinos, como a Lagoa Salgada, além de mananciais termais (Srivastava, 2000).

Os biolaminóides, objeto deste trabalho, compõem o primeiro estágio de desenvolvimento estromatolítico e o primeiro substrato fixo, necessário para o desenvolvimento do estromatólito (Silva e Silva, 2002). E, assim como os estromatólitos, esse estágio também é caracterizado por estruturas organosedimentares laminadas "verticalmente" que se desenvolvem em superfícies coesas e se caracterizam principalmente por um grupo de microorganismos, as cianobactérias, bactérias incolores, bactérias púrpuras e bactérias redutoras de sulfato, além de possuírem, freqüentemente, gradientes saturados de oxigênio e sulfeto (Van Gemerdem, 1993).

Lee (1999), em sua obra, definiu os biolaminóides como um depósito de cianobactérias resultante da captação e coalescência do sedimento e da precipitação do carbonato de cálcio. Segundo Golubic (1973), as cianobactérias que dominam estas estruturas adquiriram especiação e adaptação devido à pressão do meio, garantindo a sobrevivência em ambientes inóspitos com elevadas taxas de salinidade e alcalinidade. Os biolaminóides são encontrados geralmente em locais hipersalinos e em ambientes cujas condições severas são restritivas ao desenvolvimento dos metazoários pastadores (Horodyski et al.,1977).

A compreensão do crescimento dos biolaminóides contemporâneos tem aplicação para interpretar a macroestrutura, estrutura laminada e modo de formação dos biolaminóides proterozóicos preservados, assim como o estudo da biologia destas estruturas contemporâneas proporciona discernimento a respeito da microbiota formadora das construções pré-cambrianas.

Este trabalho objetivou tipificar os biolaminóides presentes na borda nordeste da Lagoa Vermelha relacionados com a formação de estromatólitos e levantar a microflora cianobacteriana presente nestas estruturas. 


\section{2 Área de estudo}

A Lagoa Vermelha está situada a aproximadamente $100 \mathrm{~km}$ à leste do Rio de Janeiro entre os municípios de Saquarema e Araruama na planície costeira possuindo as seguintes coordenadas $22^{\circ} 55^{\prime} 39^{\prime \prime}$ e $22^{\circ} 56^{\prime} 06^{\prime \prime}$ de latitude Sul e $42^{\circ} 21^{\prime} 29^{\prime \prime}$ e $42^{\circ} 24^{\prime} 13^{\prime \prime}$ de longitude à Oeste de Greenwich.

Suas dimensões são $4.400 \mathrm{~m}$ de comprimento, 250-850 m de largura com uma área de aproximadamente $2.400 \mathrm{~m}^{2}$ e profundidade de 0,2 a 1,7 m. Devido a pouca profundidade e a forte incidência de ventos na região, não ocorre estratificação da coluna d’água na Lagoa Vermelha (Höhn et al., 1986).

Separada do Oceano Atlântico por uma restinga de aproximadamente $350 \mathrm{~m}$ de largura e $4 \mathrm{~m}$ acima do nível do mar, a laguna foi artificialmente dividida em três compartimentos para aproveitamento pela indústria salineira.

Santelli (1988) relata que não há drenagem superficial nem rios alimentando a lagoa, tornando a variação da salinidade dependente das condições meteorológicas, uma vez que seu balanço hídrico é controlado pela precipitação, evaporação e entrada de água subterrânea de origem marinha e continental. No entanto, Primo \& Bizerril (2002) afirmam que a Lagoa Vermelha possui ligação com Lagoa de Araruama por canais que passam entre os tanques de secagem das salinas.

O fundo da lagoa é coberto por um tapete algal, laminado, gelatinoso, de 2 a $8 \mathrm{~cm}$ de espessura. Em épocas de seca, a lagoa diminui sua massa de água, como conseqüência da grande evaporação, e suas águas sofrem um recuo de até $6 \mathrm{~m}$ em relação á época chuvosa, expondo o tapete algal nestas regiões (Santelli, 1988). De acordo com Höhn et al.(1986), os biolaminóides se desenvolvem bem no ambiente da Lagoa Vermelha e se distribuem ao longo da margem indo até 30-40 metros para dentro da laguna.

Santelli (1988) ainda relata que o sedimento da lagoa é rico em carbonatos, sendo formado quase na sua totalidade por camadas laminadas cinza claras que contêm minerais carbonáticos e camadas escuras de matéria orgânica. No topo, há acumulação de conchas de biválvios (Anomalocardia brasiliensis Gmelin, 1791) e gastrópodes.

Destaca-se ainda a presença de estromatólito em biscuit, como construções freqüentes na lagoa (Silva e Silva et al., 2004). 


\section{Materiais e Métodos}

Na margem nordeste da Lagoa Vermelha foram estabelecidos cinco pontos de coleta com distância de $30 \mathrm{~m}$ entre eles, visando abranger diferentes condições ambientais e diferentes tipos de biolaminóides.

Foi realizado um total de seis coletas e extraídas 20 amostras de biolaminóides. As amostras foram retiradas manualmente procurando manter a integridade morfológica e composicional de cada tipo de biolaminóide relacionado com a formação de estromatólitos.

Para a preservação das cianobactérias o material coletado foi fixado, no campo, em solução aquosa de formol a 4\% utilizando a água da lagoa e tamponada com bórax e levados ao laboratório para análise.

Para a caracterização da composição cianobacteriana, foram confeccionadas lâminas frescas e permanentes de cada tipo de biolaminóide. Usou-se tinta nanquim para evidenciar a bainha de mucilagem dos exemplares de cianobactérias e para as lâminas permanentes foi utilizado formol glicerinado a $25 \%$ objetivando a conservação das mesmas.

A identificação cianobacteriana foi realizada observando-se as características morfológicas clássicas e através de cinco medições feitas com o auxílio de uma ocular micrométrica, sendo estas diâmetro dos filamentos, diâmetro das colônias, diâmetro dos tricomas, espessura das bainhas, comprimento e largura das células, obtendo-se além da média os valores mínimos e máximos.

Foram aferidos no ato da coleta parâmetros físico-químicos da água e dos biolaminóides.

\section{Resultados}

Ao longo das estações de coleta foram encontrados três tipos de biolaminóides potencialmente formadores de estromatólitos, sendo eles liso, poligonal e pustular distribuídos ao longo das estações de acordo com a Tabela 1. 
Biolaminóides Calcários Holocênicos: o Caso da Lagoa Vermelha, Brasil Loreine Hermida da Silva e Silva \& Sinda Beatriz Vianna Carvalhal

\begin{tabular}{|l|c|c|c|c|c|}
\hline \multicolumn{1}{|c|}{ Biolaminóides } & Estação 1 & Estação 2 & Estação 3 & Estação 4 & Estação 5 \\
\hline Liso & $\mathrm{X}$ & $\mathrm{X}$ & $\mathrm{X}$ & $\mathrm{X}$ & $\mathrm{X}$ \\
\hline Poligonal & $\mathrm{X}$ & & & & \\
\hline Pustular & $\mathrm{X}$ & & & & \\
\hline
\end{tabular}

Tabela 1 Ocorrência dos biolaminóides ao longo das estações de coleta.

O biolaminóide liso foi encontrado em todas as estações, com localização variando de inframaré à região superior de entremarés. Apresentou-se com morfologia clássica, composta por finas laminações, distribuídas ao longo de três estratos (Figura 1) e com a seguinte composição de cianobactérias: Aphanocapsa litoralis (Hansgirg) Komárek \& Anagnostidis, 1995; Aphanocapsa salina Voronichin, 1929; Chlorogoea tuberculosa (Hansgirg) Wille 1900, Chroococcus membraninus (Meneghini) Nägeli 1849; Chroococcus minimus (Kützing) Lemmermann, 1904; Chroococcus minor (Kützing) Nägeli, 1984; Chroococcus minutus (Kützing) Nägeli, 1849; Chroococcus turgidus (Kützing) Nägeli, 1849; Cyanosarcina thalassia Anagnostidis \& Pantazidar, 1991; Gloeocapsopsis crepidinum (Thuret) Komárek, 1993; Johannesbaptista pellucida (Dickie) Taylor \& Drouet, 1938; Jaaginema subtilissimun (Kützing ex De Toni) Anagnostidis \& Komárek, 1988; Lyngbya aestuarii (Liebmann) Gomont, 1892; Lyngbya fragilis (Meneghini) Compère, 1974; Microcoleus chthonoplastes (Thuret) Gomont, 1892; Microcoleus tenerrimus Gomont, 1892; Oscillatoria limnetica Lemmermann, 1900; Phormidium chalybeum (Gomont) Anagnostidis \& Komárek, 1988; Phormidium cf. alorgei (Frémy) Anagnostidis \& Komárek,1985; Porphyrosiphon martensianus (Gomont), Anagnostidis \& Komárek 1988; Spirulina meneghiniana (Zanardini) Gomont 1892. A distribuição dos taxa ao longo dos estratos está demonstrada na Tabela 2. Dentre as famílias encontradas a família Phormidiaceae foi a que apresentou maior número de espécies no estrato verde e a família Chroococcaceae predominou nos estratos vermelho e marrom.

O biolaminóide poligonal, encontrado apenas na estação \#1 na porção superior da região de entremarés, apresentou também as características clássicas, formando fendas de ressecamento, sendo composto de finas laminações, e formando três estratos com diferentes composições microbianas (Figura 2). Nesta estrutura foi encontrado um total de vinte espécies de cianobactérias tendo a família Phormidiaceae 39\% das espécies no estrato 
Biolaminóides Calcários Holocênicos: o Caso da Lagoa Vermelha, Brasil Loreine Hermida da Silva e Silva \& Sinda Beatriz Vianna Carvalhal

superior verde e a família Chroococcaceae com 40\% e 45\% das espécies nos estratos vermelho e marrom respectivamente. Sendo a composição cianobacteriana: Aphanocapsa salina; Aphanothece conglomerata Rich, 1932; Aphanothece halophytica Frémy, 1933; Chlorogoea tuberculosa; Chroococcus microscopicus Komarová Legnerová \& Cromberg, 1994; Chroococcus minimus; Chroococcus minor; Chroococcus minutus; Chroococcus turgidus; Entophysalis conferta (Kützing) Drouet \& Daily, 1948; Johannesbaptita pellucida; Lyngbya aestuarii; Microcoleus chthonoplastes; Microcoleus tenerrimus; Oscillatoria princeps (Vaucher) Gomont 1892; Phormidium aerugineo-caeruleum (Gomont) Anagnostidis \& Komárek, 1988; Phormidium cf. allorgei; Phormidium jasorvensis Anagnostidis \& Komárek, 1988; Schizothrix friesii Gomont, 1892 e Synechococcus salinarum Komárek, 1956. A distribuição destas cianobactérias ao longo dos estratos está disposta na Tabela 3.

O biolaminóide pustular foi encontrado na estação \#1, apresentando-se como pequenas pústulas sobre o sedimento, não estratificado, de consistência gelatinosa e coloração variando entre o verde e o marrom e a seguinte composição cianobacteriana: Aphanocapsa litoralis; Aphanothece conglomerata; Aphanothece marina (Ercegovic') Komárek \& Anagnostidis, 1995; Aphanothece salina Elenking \& Danilov, 1915; Chroococcus minimus;

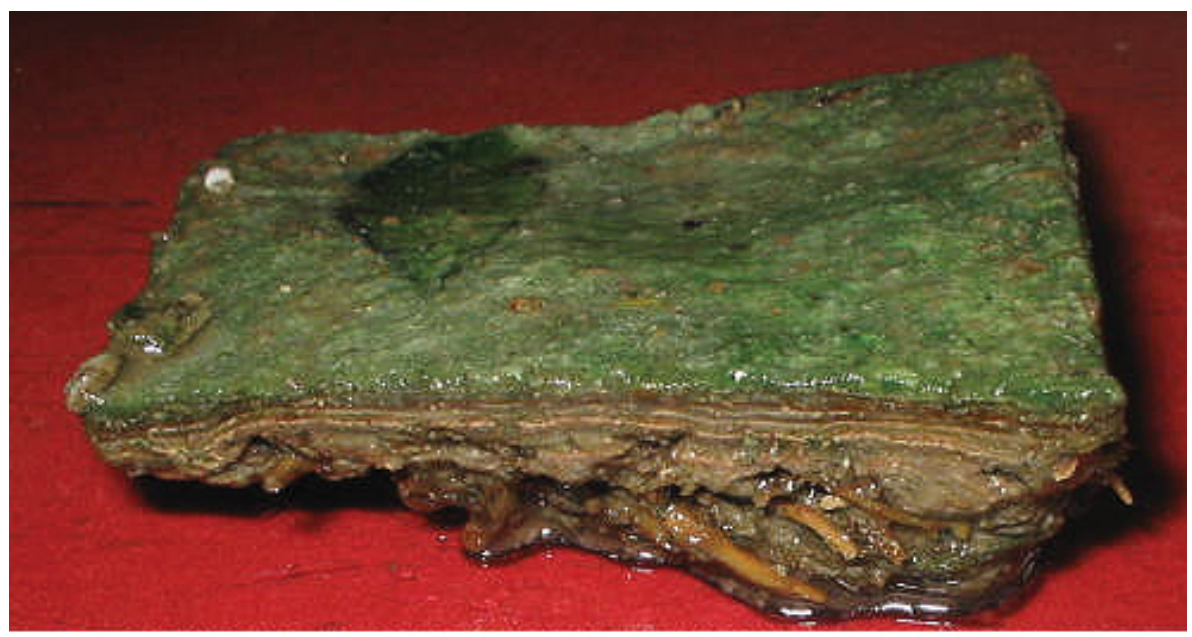

0

$1 \mathrm{~cm}$

Figura 1 Vista parcial do biolaminóide liso. 
Biolaminóides Calcários Holocênicos: o Caso da Lagoa Vermelha, Brasil Loreine Hermida da Silva e Silva \& Sinda Beatriz Vianna Carvalhal

\begin{tabular}{|l|c|c|c|}
\hline \multicolumn{1}{|c|}{ Espécie } & Estrato verde & Estrato vermelho & Estrato marrom \\
\hline Aphanocapsa litoralis & $\mathrm{X}$ & & $\mathrm{X}$ \\
\hline Aphanocpsa salina & & $\mathrm{X}$ & \\
\hline Chlorogoea tuberculosa & & $\mathrm{X}$ & $\mathrm{X}$ \\
\hline Chroococcus membraninus & & $\mathrm{X}$ & $\mathrm{X}$ \\
\hline Chroococcus minimus & & $\mathrm{X}$ & $\mathrm{X}$ \\
\hline Chroococcus minor & $\mathrm{X}$ & $\mathrm{X}$ & $\mathrm{X}$ \\
\hline Chroococcus minutus & $\mathrm{X}$ & $\mathrm{X}$ & \\
\hline Chroococcus turgidus & $\mathrm{X}$ & $\mathrm{X}$ & $\mathrm{X}$ \\
\hline Cyanosarcina thalassia & & $\mathrm{X}$ & $\mathrm{X}$ \\
\hline Gloeocapsopsis crepidinum & $\mathrm{X}$ & $\mathrm{X}$ & \\
\hline Johannesbaptita pellucida & $\mathrm{X}$ & $\mathrm{X}$ & \\
\hline Jaaginema subtilissimun & $\mathrm{X}$ & $\mathrm{X}$ & \\
\hline Lyngbya aestuarii & $\mathrm{X}$ & & \\
\hline Lyngbya fragilis & & & \\
\hline Microcoleus chthonoplastes & $\mathrm{X}$ & \\
\hline Microcoleus tenerrimus & & & \\
\hline Oscillatoria limnetica & & & \\
\hline Phormidium chalybeum & & & \\
\hline Porphyrosiphon martensianus & & & \\
\hline Phormidium cf. alorgei & & & \\
\hline Spirulina meneghiniana & & & \\
\hline
\end{tabular}

Tabela 2 Distribuição das cianobactérias ao longo dos estratos no biolaminóide liso. 
Chroococcus minor; Chroococcus minutus; Chroococcus turgidus; Chlorogoea tuberculosa; Gloeocapsa salina Hansgirg, 1893; Microcoleus chthonoplastes e Synechococcus salinarum. Dentre estas algas as famílias Synechococcaceae e Chroococcaceae apresentaram 34\% do número de espécies. A família Enthophysalidaceae apesar de englobar a espécie dominante desta esteira, a Chlorogoea tuberculosa, apresentou apenas 8\% das espécies.

\section{Conclusão}

A Lagoa Vermelha foi constatada como uma lagoa hipersalina, mantendo valores de salinidade acima de $30 \%$, e alcalina com $\mathrm{pH}$ na faixa de 8 , revelando-se um ambiente apropriado para a proliferação e domínio das cianobactérias. Foram encontrados três tipos de biolaminóides, liso, poligonal e pustular. Estes tipos são precursores dos estromatólitos, conforme afirmado por Stal (1995).

A distribuição destes biolaminóides na região litorânea na borda da lagoa é um fator dependente da umidade e da constituição cianobacteriana. A análise dos biolaminóides revelou que cada estrutura é composta por um tipo de cianobactéria principal e que esta pode ou não pertencer ao táxon que se apresenta com maior número de espécies.

Um total de 33 espécies de cianobactérias foi encontrado nos biolaminóides. Os lisos e poligonais se apresentaram estratificados, compostos

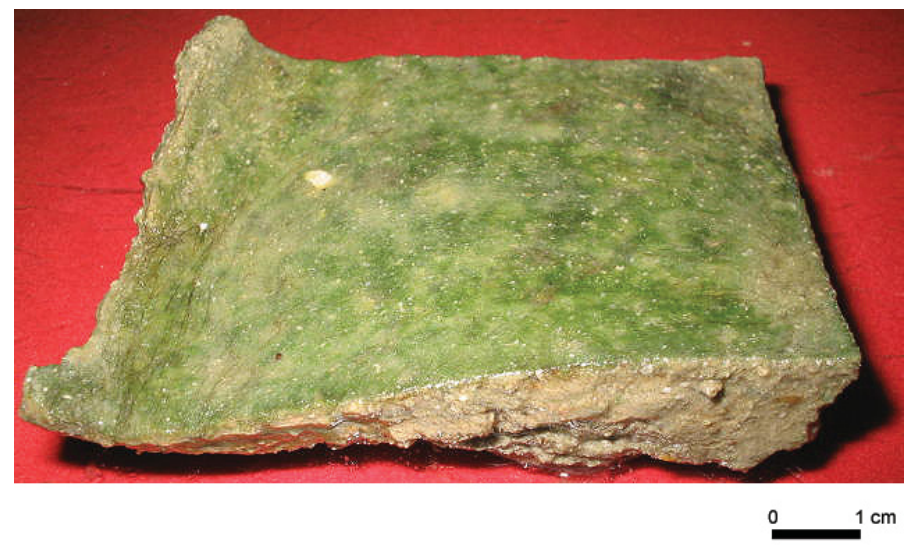

Figura 2 Biolaminóide poligonal. 
Biolaminóides Calcários Holocênicos: o Caso da Lagoa Vermelha, Brasil Loreine Hermida da Silva e Silva \& Sinda Beatriz Vianna Carvalhal

por diversos estratos, sendo o mais superficial de coloração verde, um intermediário vermelho e um marrom mais profundo.

\begin{tabular}{|l|c|c|c|}
\hline \multicolumn{1}{|c|}{ Espécie } & Estrato verde & Estrato vermelho & Estrato marrom \\
\hline Aphanocapsa salina & $\mathrm{X}$ & $\mathrm{X}$ & \\
\hline Aphanothece halophytica & $\mathrm{X}$ & & \\
\hline Aphanothece conglomerata & & $\mathrm{X}$ & \\
\hline Chlorogoea tuberculosa & & $\mathrm{X}$ & $\mathrm{X}$ \\
\hline Chroococcus microscopicus & & $\mathrm{X}$ & $\mathrm{X}$ \\
\hline Chroococcus minimus & & $\mathrm{X}$ & $\mathrm{X}$ \\
\hline Chroococcus minor & & $\mathrm{X}$ & $\mathrm{X}$ \\
\hline Chroococcus minutus & $\mathrm{X}$ & $\mathrm{X}$ & $\mathrm{X}$ \\
\hline Chroococcus turgidus & $\mathrm{X}$ & $\mathrm{X}$ & \\
\hline Entophysalis conferta & $\mathrm{X}$ & & $\mathrm{X}$ \\
\hline Johannesbaptista pellucida & & & \\
\hline Lyngbya aestuarii & $\mathrm{X}$ & & \\
\hline Microcoleus chthonoplastes & & & \\
\hline Microcoleus tenerrimus & & & \\
\hline Oscillatoria princeps & & & \\
\hline Phormidium aerugineocaeruleum & $\mathrm{X}$ & \\
\hline Phormidium cf. allorgei & & & \\
\hline Phormidium jasorvensis & & & \\
\hline Schizothrix friesii & & & \\
\hline Synechococcus salinarum & & & \\
\hline
\end{tabular}

Tabela 3 Distribuição das cianobactérias ao longo dos estratos no biolaminóide poligonal. 
Biolaminóides Calcários Holocênicos: o Caso da Lagoa Vermelha, Brasil Loreine Hermida da Silva e Silva \& Sinda Beatriz Vianna Carvalhal

O biolaminóide liso revelou ser formado principalmente por Lyngbya aestuarii, apresentando em suas estratificações a predominância da família Phormidiaceae no estrato verde e a família Chroococaceae nos estratos vermelho e marrom.

O biolaminóide poligonal exibiu-se composto por Microcoleus chthonoplastes, apresentando em suas estratificações a predominância da família Phormidiaceae no estrato verde e a família Chroococaceae nos estratos vermelho e marrom.

O biolaminóide pustular caracterizou-se pela presença da cianobactéria Chlorogoea tuberculosa contrariando o trabalho de Hoffmann (1974) em que a microalga dominante é a Entophysalis major. Ressalta-se aqui que as duas espécies de cianobactérias pertencem à mesma subfamília Entophysalidoideae, postulando-se então a hipótese de que as características morfológicas deste biolaminóide devem estar relacionadas às características da subfamília a que pertencem estas cianobactérias como tipo de talo (colonial) e organização celular dentro das colônias.

\section{Referências}

Golubic, S. 1973. The relationship between blue green algae and carbonate deposits. In: CARR, N.G. \& WHITTON, B.A. (eds.). The Biology of Blue green algae. Oxford Blackwell Scientific Publications, p.434-472.

Hoffmann, P.F. 1974. Shalow and deepwater stromatolites in Lower Proterozoic platform-to-basin facies change, Great Slave Lake, Canada. American Associated Petroleum Geologists Bulletin, 58: 856-867.

Hönn, A., Tobschal, H.J. \& Maddock, J.E.L. 1986. Biogeochemistry of a hipersaline lagoon east of Rio de Janeiro, Brazil. The Science of the total environment. Amsterdam Eselvier Science, 58: 175-185.

Horodyski, R.J., Bloeser, B. \& Vonder Haar, S. 1977. Laminated algal mats from a coastal lagoon, Laguna Mormona, Baja California, Mexico. Journal of Sedimentary Petrology, 47 (2): 680-696.

Lee, R.E. 1999. Phycology. Cambridge University Press, 614p.

Primo, P.B.S. \& Bizerril, C.R.S.F. 2002. Lagoa de Araruama: perfil ambiental do maior ecossistema lagunar do mundo. SEMADS, Rio de Janeiro, 160 p. (Série Projeto Planágua)

Santelli, R. C. L. 1988. Estudos de isótopos estáveis em sedimentos carbonáticos da Lagoa Vermelha - RJ. Programa de Pós-graduação em Química, Pontifícia Universidade Católica do Rio de Janeiro, Tese de Doutorado, $95 \mathrm{p}$. 
Silva e Silva, L. H. 2002. Contribuição ao conhecimento da composição microbiana e química das estruturas estromatolíticas da Lagoa Salgada, Quaternário do Rio de Janeiro, Brasil. Programa de Pós-graduação em Geologia, Universidade Federal do Rio de Janeiro, Tese de Doutorado, 176 p.

Silva e Silva, L. H.; Senra, T.C.L.M.; Faruolo T.C.L.M; Carvalhal, S. B. V.; Alves, S. A. P. M. N.; Damazio, C. M.; Shimizu, V. T. A.; Santos, R.C \& Iespa, A.A.C. 2004. Composição paleobiológica das construções estromatolíticas da Lagoa Vermelha RJ, Brasil. Revista Brasileira de Paleontologia. 7 (2): 193-198.

Srivastava, N.K. 2000. Estromatólito. In: CARVALHO, I. S. (ed.). Paleontologia. Rio de Janeiro, Interciência, p.119-134.

Stal, J.L. 1995. Physiological ecology of cyanobacteria in microbial mats and others comunities. New Phytologist. 131: 1-32.

Van Gemerdem, H. 1993. Microbial mats: a joint venture. Marine Geology, 133: 3-25. 